\title{
AN ELEMENTARY THEORY OF THE CATEGORY OF TOPOLOGICAL SPACES $\left({ }^{1}\right)$
}

\author{
BY \\ DANA I. SCHLOMIUK
}

\begin{abstract}
An elementary system of axioms was given by F. W. Lawvere for the category of sets and mappings. The purpose of this paper is to provide a finite number of elementary axioms for the category of topological spaces and continuous mappings and to prove that any model of these axioms is equivalent to "the category of topological spaces" constructed over some model of Lawvere's axioms. Furthermore, we prove that any complete category, model of the given axioms is equivalent to the category of topological spaces.
\end{abstract}

1. Introduction. We shall list a finite number of elementary axioms which hold in the category $\boldsymbol{T}$ of topological spaces and continuous mappings. It will be shown that any model of the given elementary axioms is equivalent to "the category of topological spaces" constructed over some model of Lawvere's axioms [8].

In addition to the above result, which is of purely elementary character, we prove that any complete category, model of the author's axioms is equivalent to $\boldsymbol{T}$. This is an analogue for $\boldsymbol{T}$ of Lawvere's result for the category of sets [8].

Apart from providing a characterization of the category of topological spaces and continuous mappings (in the sense mentioned above), the set of elementary axioms could be used in principle for developing topology independently of any set-theoretical foundation.

In order to state the axioms we use the language of category theory. The definition of an abstract category in the sense of Eilenberg-Maclane [2] can be formalized [9] in a language with one sort of variable symbols (the mappings), two unary function symbols (domain and codomain) and one ternary relation symbol (composition). The axioms which form this definition are all elementary, i.e. all quantifiers range over individual variables.

The axioms for $\boldsymbol{T}$ are stated in the language of category theory augmented by an individual constant $o$ which makes it possible to define the notion of "open subspace".

Received by the editors April 29, 1969.

AMS Subject Classifications. Primary 1810, 0250, 5410.

Key Words and Phrases. Extremal monomorphism, regular monomorphism, regular image, projective generator, suitable topology, exponentiation axiom, Lawvere's axiom of infinity.

( $\left.{ }^{1}\right)$ The material in this article is based on the author's doctoral dissertation (McGill University 1967).

Copyright (C) 1970, American Mathematical Society 
All but one of the elementary axioms are simple properties of $\boldsymbol{T}$. The last elementary axiom is more complex in character. Intuitively it says that for every set and interior operation on it there exists an object in the category which "corresponds" to it. When this axiom is deleted a full imbedding into the category of topological spaces is still obtained.

The author wishes to thank Professor J. Lambek and also B. Rattray for help and constant encouragement. Special thanks are due to F. W. Lawvere for enlightening comments, suggestions and stimulating conversations. Thanks are also due to the referee for suggestions.

2. Notation and terminology. We shall use the notation of Lawvere. Objects, i.e. maps which are also domains or codomains will be denoted by capital letters. $A \stackrel{f}{\rightarrow} B$ stands for: " $f$ is a map whose domain is $A$ and whose codomain is $B$ ". The result of the composition of the maps $A \stackrel{f}{\rightarrow} B$ and $B \stackrel{g}{\rightarrow} C$ will be denoted by $f g$. The notations $\left({ }^{2}\right) k=\mathrm{Eq}(f, g)$ and $q=\operatorname{Coeq}(f, g)$ mean respectively $k$ is an equalizer and $q$ is a coequalizer of $f$ with $g . A=A_{1}+A_{2}, A=A_{1} \times A_{2}$ mean respectively that there exist injections $i_{1}, i_{2}$ and projections $p_{1}, p_{2}$ such that $A, i_{1}, i_{2}$ form a sum and $A, p_{1}, p_{2}$ form a product of $A_{1}$ and $A_{2}$. The map into $A_{1} \times A_{2}$ determined by $X \stackrel{f}{\rightarrow} A_{1}$ and $X \stackrel{g}{\rightarrow} A_{2}$ will be denoted by $\langle f, g\rangle$. A map which is an equalizer (coequalizer) of a pair of mappings is called a regular monomorphism (regular epimorphism). An object $P$ is projective if and only if for every epimorphism $A \stackrel{f}{\rightarrow} B$ and map $P \stackrel{g}{\rightarrow} B$, there exists a map $P \stackrel{h}{\rightarrow} A$ such that $g=h f$. An object $G$ is a generator if for every pair of maps $A \stackrel{f}{\rightarrow} B, A \stackrel{g}{\rightarrow} B$ such that $f \neq g$ there exists a map $G \stackrel{h}{\rightarrow} A$ such that $h f \neq h g$.

By a complete category we mean a category which has small limits and colimits. The category of sets and functions will be denoted by $\boldsymbol{S}$ and the category of topological spaces and continuous mappings by $\boldsymbol{T}$.

3. An elementary theory of the category of topological spaces. In this section we state the elementary axioms for $\boldsymbol{T}$ and we prove some theorems of the resulting elementary theory.

The first group of axioms is formed by those which define an abstract category. We may refer to the conjunction of these axioms as Axiom 0.

Another group is a fragment of Lawvere's elementary system for the category of sets [8].

AxIom 1. There exists a unique initial and a terminal object; every pair of objects has a product and a sum; every pair of mappings has an equalizer and a coequalizer.

We shall denote by 0 the initial object.

The above axiom implies the existence of pull-backs and push-outs. Also, for every mapping $A \stackrel{f}{\rightarrow} B$ we can carry out the following construction:

$\left({ }^{2}\right)$ The equality here is actually an abuse of language. 


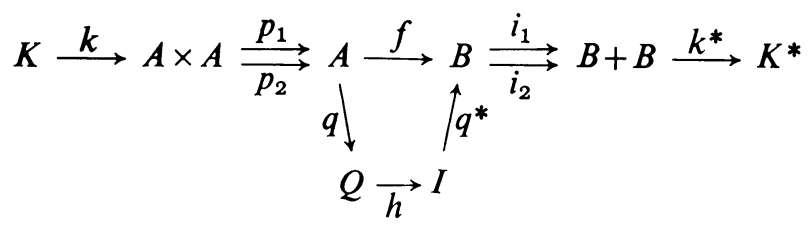

where $k=\operatorname{Eq}\left(p_{1} f, p_{2} f\right) ; k^{*}=\operatorname{Coeq}\left(f i_{1}, f i_{2}\right) ; q=\operatorname{Coeq}\left(k p_{1}, k p_{2}\right) ; q^{*}=\operatorname{Eq}\left(i_{1} k^{*}, i_{2} k^{*}\right)$; and $h$ is the unique mapping for which the above diagram is commutative.

The mappings $q, q^{*}$ are called respectively, the regular coimage of $f$ and the regular image of $f$. We write $q=\operatorname{Coim} f$ and $q^{*}=\operatorname{Im} f$.

For expository purposes we shall make two temporary assumptions.

Assumption 1. There exists a unique terminal object which we denote by 1 .

Definition 1. $x$ is an element of $A$, or $x \in A$, if and only if $\stackrel{x}{\rightarrow} A$.

Assumption 2. There exists an object with more than one element.

COROLlary. 0 has no element.

Axıom 2. The object 1 is a projective generator.

The fact that 1 is projective means that the epimorphisms are onto mappings and this holds in $\boldsymbol{T}$.

The following proposition is an immediate consequence of Axiom 2.

Proposition 1. Let $f$ be a mapping with domain $A$ and codomain $B$. Then:

(i) $f$ is an epimorphism if and only if $f$ is surjective, i.e. for every $x \in B$ there exists $y \in A$ such that $y f=x$.

(ii) $f$ is a monomorphism if and only if $f$ is injective, i.e. for every pair of elements $x, y$ of $A$ such that $x \neq y$, we have $x f \neq y f$.

DefinItion 2. A mapping $f$ is a bijection if and only if it is both an epimorphism and a monomorphism.

Definition 3. A mapping $A \stackrel{f}{\rightarrow} B$ is called a constant mapping if there exists $u \in B$ such that $A \stackrel{f}{\rightarrow} B=A \rightarrow 1 \stackrel{u}{\rightarrow} B$.

Axiom 3. Every element of a sum $A+B$ can be factored through one of the injections $i_{A}, i_{B}$ i.e. if $x \in A+B$ there exists a map $t$ such that $x=t i_{A}$ or $x=t i_{B}$.

Definition 4. $2=1+1$.

The following propositions are immediate consequences of the axioms and assumptions.

Proposition 2. The two injections $1 \stackrel{i_{0}}{\rightarrow} 2$ and $1 \stackrel{i_{1}}{\rightarrow} 2$ are different and they are the only elements of 2 .

Proposition 3. If $x \in A+B$, then $x$ cannot be factored through both injections $i_{A}$ and $i_{B}$, i.e. at most one of the equations $x=t i_{A}$ and $x=t i_{B}$ has a solution $t$.

The next group of axioms involve the notion of discrete space which we define as follows: 
Definition 5. An object $A$ is called a discrete space if for every $x \in A$ there exists a mapping $A \stackrel{f_{x}}{\rightarrow} 2$ such that for every $y \in A, y \neq x$, we have $x f_{x} \neq y f_{x}$.

Clearly the objects 0,1 are discrete spaces. Also 2 is discrete since 2 has only two elements and the identity on 2 satisfies the property of $f$ in the above definition for each one of them.

The following weaker form of the axiom of choice used by Lawvere for $\boldsymbol{S}$ clearly holds in $\boldsymbol{T}$.

Axıom 4. For every map $A \stackrel{f}{\rightarrow} B$ such that $A \neq 0$ and $B$ is a discrete space, there exists a map $g$ such that $f g f=f$.

Proposition 4. Every nonzero object $A$ has elements and the mapping $A \stackrel{t}{\rightarrow} 1$ is an epimorphism.

Proposition 5. If $A$ and $B$ are discrete and $A \stackrel{f}{\rightarrow} B$ is a bijection then $f$ is an isomorphism.

Proof. If $A=0$, by Proposition $4, B=0$ since $f$ is an epimorphism. If $A \neq 0$, by Axiom 4 there is a $g$ such that $f g f=f$ and since $f$ is a bijection $f g=A$ and $g f=B$.

Axıom 5. For every object $A$, there exists a discrete space $|A|$ together with a mapping $|A| \stackrel{t_{A}}{\rightarrow} A$ such that for every discrete space $B$ and mapping $B \stackrel{f}{\rightarrow} A$, there exists a unique mapping $h$ for which the diagram below is commutative.

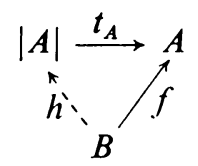

$|A|$ is defined up to isomorphism and the operation || is functorial. (The mapping $|A| \stackrel{t_{A}}{\rightarrow} A$ is a reflection [9] of $A$ into the discrete spaces, and the functor | $\mid$ is an adjoint of the inclusion functor.)

If $A$ is a discrete space, the mapping $A$ satisfies the property of $t_{A}$ in the above axiom and hence we write $|A|=A=t_{A}$.

Proposition 6. The mapping $|A| \stackrel{t_{A}}{\rightarrow} A$ is a bijection.

Proof. By Proposition 2, in order to show that $t_{A}$ is a monomorphism, it is sufficient to prove that for every pair $x, y$ of elements of $A$ such that $x t_{A}=y t_{A}$, we have $x=y$. This follows from Axiom 5 since 1 is discrete and hence the mapping $x t_{A}\left(=y t_{A}\right)$ must factor uniquely through $t_{A} \cdot t_{A}$ is also an epimorphism. This follows from Proposition 1, the fact that 1 is discrete and Axiom 5.

COROLlaRY. The functor | | is faithful, i.e. for every pair of mappings $A \stackrel{f}{\rightarrow} B$, $A \stackrel{g}{\rightarrow} B$ such that $|f|=|g|$, we have $f=g$.

Proof. If $|f|=|g|$, then $t_{A} f=|f| t_{B}=|g| t_{B}=t_{A} g$ and since $t_{A}$ is an epimorphism, $f=g$. 
Proposition 7. A mapping $f$ is a monomorphism (epimorphism) if and only if $|f|$ is a monomorphism (epimorphism).

Proof. Since | | is faithful, it reflects monomorphisms and epimorphisms, i.e. if $|f|$ is mono (epi) then so is $f$. Since $|\quad|$ is a right adjoint functor, it must preserve monomorphisms. This follows from the fact that a right adjoint functor preserves pull-back diagrams and $f$ is a monomorphism if and only if the diagram below is a pull-back.

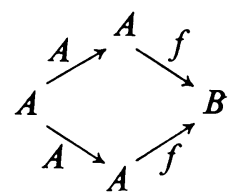

It remains to show that | preserves epimorphisms. Suppose $f$ is an epimorphism. By Proposition 2, in order to show that $|f|$ is epi it will suffice to prove that $|f|$ is surjective. Both $t_{A}$ and $f$ are surjective and hence for every $y \in|B|$, there exists $x \in|A|$ such that $x t_{A} f=y t_{B} \in B$. Therefore $x|f| t_{B}=x t_{A} f=y t_{B}$ and since $t_{B}$ is a monomorphism $x|f|=y$. Q.E.D.

COROllary. For every discrete space $D$ and bijection $D \stackrel{d}{\rightarrow} A$ we have $d \cong t_{A}$.

Proof. Since $D$ is discrete, $D=|D|=t_{D}$ and hence $\stackrel{|| d \mid}{\rightarrow}|A|$. The above proposition together with the fact that $d$ is a bijection implies that $|d|$ is a bijection. But $D$ and $|A|$ are discrete and hence by Proposition $5,|d|$ is an isomorphism. Q.E.D.

In the following theorem we study some properties of the discrete spaces.

THEOREM 1. (a) If $A \stackrel{m}{\rightarrow} B$ is a monomorphism and $B$ is discrete, then $A$ is discrete.

(b) If $A$ and $B$ are discrete, then $A+B$ is discrete.

(c) If $B \stackrel{h}{\rightarrow} Q$ is a regular epimorphism and $B$ is discrete, then $Q$ is discrete.

Proof. (a) Suppose $x \in A$ and consider $u=x m$. Then $u \in B$ and since $B$ is discrete, there exists a mapping $B \stackrel{f_{u}}{\longrightarrow} 2$ such that for every $v \in B, v \neq u$ we have $v f_{u} \neq u f_{u}$. Let $f_{x}=m f_{u}$. If $y \in A$ and $y \neq x$, since $m$ is a monomorphism, we have $y m \neq x m$ $(=u)$ and hence $y f_{x}=y m f_{u} \neq x m f_{u}=x f_{x}$. Q.E.D.

(b) Since $A$ and $B$ are discrete, we have $A=|A|=t_{A}, B=|B|=t_{B}$. Consider the pair $|A+B|, t_{A+B}$ defined by Axiom 5 .

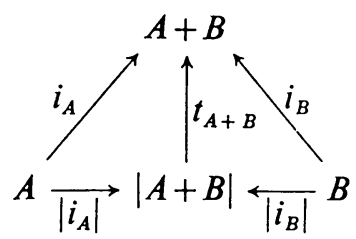

Since $A+B$ is a sum, there exists a unique mapping $A+B \stackrel{u}{\rightarrow}|A+B|$ such that 
$i_{A} u=\left|i_{A}\right|, i_{B} u=\left|i_{B}\right|$ and hence $i_{A} u t_{A+B}=i_{A}, i_{B} u t_{A+B}=i_{B}$. Therefore, $u t_{A+B}=A+B$ which implies that $u$ is a monomorphism. By (a) since $|A+B|$ is discrete, we have that $A+B$ is discrete.

(c) Suppose

$$
h=\operatorname{Coeq}(f, g) \text { where } A \stackrel{f}{\stackrel{f}{\longrightarrow}} B .
$$

Since $B$ is discrete, by Axiom 6 there exists a unique mapping $q$ for which the diagram below is commutative.

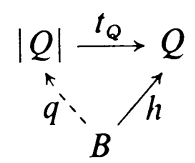

Then $f q t_{Q}=f h=g h=g q t_{Q}$ and since $t_{Q}$ is a monomorphism it follows that $f q=g q$. This implies the existence of a unique mapping $Q \stackrel{u}{\rightarrow}|Q|$ such that $h u=q$. Therefore, $h u t_{Q}=q t_{Q}=h$ and since $h$ is an epimorphism $u t_{Q}=Q$. It follows that $u$ is a monomorphism and since $|Q|$ is discrete, by (a), $Q$ is discrete. Q.E.D.

Our next axiom refers to function spaces. Given topological spaces $A$ and $B$, a topology on the set $C(A, B)$ of continuous mappings from $A$ to $B$ is called suitable [13], if for every topological space $X$, a function $X \stackrel{f}{\rightarrow} C(A, B)$ is continuous if and only if the function

$$
A \times X \stackrel{A \times f}{\longrightarrow} A \times C(A, B) \stackrel{e}{\longrightarrow} B
$$

is continuous, $e$ being the evaluation mapping i.e. for every $a \in A$ and $g \in C(A, B)$, $e(a, g)=g(a)$. There does not always exist a suitable topology on $C(A, B)$ [13], [1], [3]. If $A$ is a locally compact Hausdorff space, then the compact open topology on $C(A, B)$ is suitable. This follows immediately [13] by using results of Ahrens and Fox [1], [3]. In particular if $A$ is a discrete space, there exists a suitable topology on $C(A, B)$ and hence the following axiom holds in $T$.

Axiom 6. For every discrete space $A$ and object $B$, there exists an object $B^{A}$ and a mapping $A \times B^{A} \stackrel{e}{\rightarrow} B$ (called evaluation) such that for every object $X$ and mapping $A \times X \stackrel{f}{\rightarrow} B$ there exists a unique mapping $X \stackrel{h}{\rightarrow} B^{A}$ such that the diagram below is commutative.

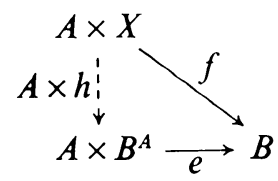

We shall denote by $f^{*}$ the unique mapping for which the following diagram commutes. 


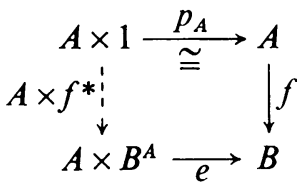

The operation $B^{A}$ is functorial and if $A_{1}, A_{2}$ are discrete spaces, $A_{2} \stackrel{f}{\rightarrow} A_{1}$, $B_{1} \stackrel{g}{\rightarrow} B_{2}$ and $A_{1} \stackrel{u}{\rightarrow} B_{1}$ then $u^{*} g^{f}=(f u g)^{*}$.

The following property is a consequence of the above axiom and the preservation properties of adjoint functors.

Proposition 8. If $A$ is discrete then $A \times(B+C) \cong A \times B+A \times C$ and $(B \times C)^{A}$ $\cong B^{A} \times C^{A}$.

In particular $2 \times 2=2 \times(1+1)=2+2$ and the two injections are $2=2 \times 1 \stackrel{2 \times i_{0}}{\longrightarrow}$ $2 \times 2$ and $2=2 \times 1 \stackrel{2 \times i_{1}}{\longrightarrow} 2 \times 2, i_{0}, i_{1}$ being the injections into 2 .

We denote by $\wedge$ the map which makes the diagram below commutative $\left({ }^{3}\right)$.

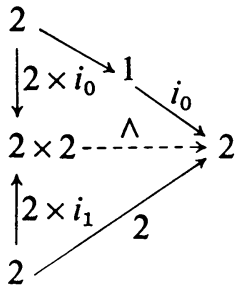

Proposition 9. The product of two discrete spaces is discrete.

Proof. If $x \in A \times B$ then $x=\langle a, b\rangle$ for some $a \in A$ and $b \in B$. If $A$ and $B$ are discrete spaces then there exists mappings $A \stackrel{f_{a}}{\rightarrow} 2$ and $B \stackrel{f_{b}}{\rightarrow} 2$ separating $a$ in $A$ and $b$ in $B$. We may assume $a f a=i_{1}=b f b$. Then the map

$$
A \times B \stackrel{\langle f a, f b\rangle \wedge}{\longrightarrow} 2
$$

separates $x$ in $A \times B$, i.e. if $f_{x}=\langle f a, f b\rangle \wedge$ then for every $y \in A \times B, y \neq x$ we have $x f_{x} \neq y f_{x}$.

AXIOM 7 (LAWVERE'S AXIOM OF INFINITY). There exists an object $N$ together with mappings $1 \stackrel{z}{\rightarrow} N$ and $N \stackrel{s}{\rightarrow} N$ such that for every mapping $1 \stackrel{x_{0}}{\rightarrow} X$ and $X \stackrel{r}{\rightarrow} X$ there exists a unique $x$ making the diagram below commutative.

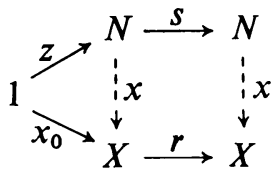

$\left.{ }^{3}\right)(\wedge$ corresponds to the truth-table of the conjuction.) 
Proposition 10. The object $N$ is discrete.

Proof. We apply Axiom 7 to $X=|N|, r=|s|$ and $x_{0}=|z|$. We obtain $x t_{N}=N$ and hence, by Theorem 1(a), $N$ is discrete.

THEOREM SCHEMA. If $\Phi$ is a theorem of the elementary theory of the category of sets [8] and $\bar{\Phi}$ is obtained from $\Phi$ by replacing "object" with "discrete object", then $\bar{\Phi}$ is a theorem in our system.

Proof. It suffices to prove the theorem for the case $\Phi$ is an axiom. In the case of Axiom 1 this follows from Theorem 1 and the fact that the functor || , being a right adjoint, preserves products and equalizers. In the case of the axiom of exponentiation, if $A$ and $B$ are discrete spaces and $e, B^{A}$ are given by Axiom 6 , then $|e|$ and $\left|B^{A}\right|$ satisfy the universal property for discrete spaces $X$. This follows by applying $\mid$ to the diagram in Axiom 6 and remarking that $t_{A \times X}=A \times X$ since by Proposition $9, A \times X$ is discrete. The fact is clear for all the remaining axioms.

For the development of our theory we shall need the following propositions obtained from the above schema.

Proposition 11. If $f$ is a mapping with discrete domain and codomain and $f=q h q^{*}$ is the standard factorization of $f$, then $h$ is an isomorphism.

The above proposition follows from the theorem schema by taking $\Phi$ to be the factorization theorem in Lawvere's system and observing that $q$ and $q^{*}$ are actually the regular discrete coimage, respectively image of $f$.

Proposition 12. Every monomorphism $A \stackrel{m}{\rightarrow} D$ into a discrete space $D$ has $a$ complement, i.e. there exists a monomorphism $A^{\prime} \stackrel{m^{\prime}}{\rightarrow} D$ such that $D, m, m^{\prime}$ form a sum. Moreover $m$ has a characteristic function $D^{\phi_{m}} \rightarrow$ 2, i.e. $m=\mathrm{Eq}\left(\phi_{m}, D \rightarrow 1 \stackrel{i_{1}}{\rightarrow} 2\right)\left({ }^{4}\right)$.

In $\boldsymbol{T}$ a subspace of a topological space $(X, \tau)$ is a topological space $\left(A, \tau_{A}\right)$ where $A$ is a subset of $X$ and $\tau_{A}$ is the topology induced by $\tau$ on $A$. The inclusion mapping $i$ is continuous and clearly the map $\left(A, \tau_{A}\right) \stackrel{\mathfrak{b}}{\rightarrow}(X, \tau)$ is a monomorphism in $\boldsymbol{T}$. Hence, to every subspace of $(X, \tau)$ we can associate a monomorphism defined on it and with codomain $(X, \tau)$. Not every monomorphism into $(X, \tau)$ has as domain a subspace (or a space isomorphic to a subspace) of $(X, \tau)$. For example consider the space $(A, \delta)$ where $\delta$ is the discrete topology on $A$. If we denote by $j$ the inclusion map $A \subseteq X$, then $j$ is continuous, the map $(A, \delta) \stackrel{j}{\rightarrow}(X, \tau)$ is a monomorphism in $T$ but $(A, \delta)$ is not necessarily a subspace of $(X, \tau)$. This shows that the notion of monomorphism is not restrictive enough to define "subspace" in our system. The notion which appears to be suitable for this purpose is that of extremal monomorphism and was introduced by Isbell [5].

Definition 6 (ISBELL). A monomorphism $f$ is called extremal if for every factorization $f=e m$ where $e$ is an epimorphism and $m$ a monomorphism, $e$ must be an isomorphism.

$\left.{ }^{4}\right)$ Assuming that a fixed labelling of the injections into 2 has been chosen, i.e. $1 \underset{i_{1}}{\stackrel{10}{\longrightarrow}} 2$. 
It can easily be seen that in $\boldsymbol{T}$ the monomorphisms associated with subspaces are extremal and every domain of an extremal monomorphism is isomorphic to a subspace.

The following proposition holds in every category [5].

Proposition 13. A regular monomorphism is extremal.

The converse of this proposition also holds in $T$. Indeed, if $X \stackrel{m}{\rightarrow} Y$ is an extremal monomorphism in $\boldsymbol{T}$, then $m \cong m(x) \stackrel{i}{\rightarrow} Y$ where $i$ is the inclusion. Clearly $i=$ Eq $(\phi, \psi)$ where $Y \stackrel{\oplus}{\rightarrow} J$ and $Y \stackrel{\psi}{\rightarrow} J, J$ being the space with two points 0,1 and two open sets, $\phi$ the characteristic function of $m(x)$ and $\psi(y)=1$ for all $y \in Y$. Hence, in $\boldsymbol{T}$, the notions of regular monomorphism and extremal monomorphism coincide. This justifies the following definition.

Definition 7. A mapping $a$ is a subspace of an object $X$, written $a \subseteq X$, if $a$ is a regular monomorphism whose codomain is $X$.

Clearly $X, 0 \rightarrow X$ and every $x \in X$ are subspaces of $X$. Also for every discrete space $D$ and monomorphism $A \stackrel{m}{\rightarrow} D, m$ is a subspace of $D$.

Definition 8. A mapping $a$ is a subspace of a mapping $b$, written $a \subseteq b$, if $a$ and $b$ have the same codomain $X, a \subseteq X, b \subseteq X$ and there exists a mapping $h$ such that the diagram below commutes.

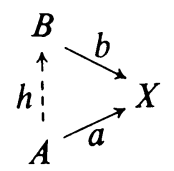

The use of $\subseteq$ for a more general situation creates no ambiguity. If $a \subseteq b$ and $a$ is an element we write $a \in b$.

Definition 9. If $a \subseteq X, b \subseteq X$ and the diagram below is a pull-back diagram,<smiles>[X]C1[Y][Te]P[P][P]1</smiles>

then the mapping $u a(=v b)$ is called the intersection of $a$ with $b$ and is denoted by $a \cap b$.

The following theorem holds in any category with finite products and equalizers.

THEOREM 2. The intersection of two regular monomorphisms is a regular monomorphism. In fact, if $a=\mathrm{Eq}\left(a_{1}, a_{2}\right)$ and $b=\mathrm{Eq}\left(b_{1}, b_{2}\right)$ then

$$
a \cap b=\operatorname{Eq}\left(\left\langle a_{1}, b_{1}\right\rangle,\left\langle a_{2}, b_{2}\right\rangle\right) \text {. }
$$

Proof. Suppose $a=\mathrm{Eq}\left(a_{1}, a_{2}\right)$ and $b=\mathrm{Eq}\left(b_{1}, b_{2}\right)$ and consider

$$
k=\mathrm{Eq}\left(\left\langle a_{1}, b_{1}\right\rangle,\left\langle a_{2}, b_{2}\right\rangle\right) \text {. }
$$

We shall prove that $k=a \cap b$. 
For this we need two mappings $u$ and $v$ such that $u, v, a, b$ form a pull-back diagram and $k=u a$. Let $f_{1}=\left\langle a_{1}, b_{1}\right\rangle$ and $f_{2}=\left\langle a_{2}, b_{2}\right\rangle$.

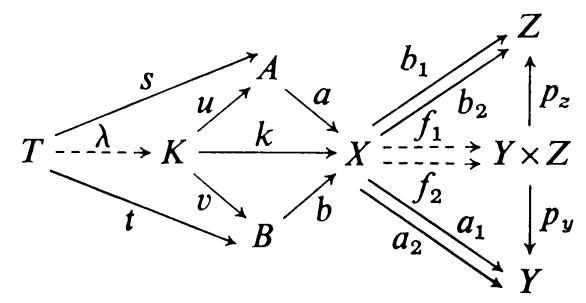

Since $k a_{1}=k f_{1} p_{y}=k f_{2} p_{y}=k a_{2}$ there exists a mapping $u$ such that $k=u a$. Similarly $k b_{1}=k b_{2}$ and hence $k=v b$ for some $v$. If for two mappings $T \stackrel{s}{\rightarrow} A$ and $T \stackrel{t}{\rightarrow} B$ we have that $s a=t b$, then $s a f_{1} p_{y}=s a a_{1}=s a a_{2}=s a f_{2} p_{y}$ and $s a f_{1} p_{z}=s a b_{1}=t b b_{1}=t b b_{2}$ $=s a b_{2}=s a f_{2} p_{z}$. Since $Y \times Z, p_{y}, p_{z}$ form a product it follows that $s a f_{1}=s a f_{2}$. But $k=\operatorname{Eq}\left(f_{1}, f_{2}\right)$. Therefore there exists a unique mapping $\lambda$ such that $s a=\lambda k$. Hence $s a=\lambda u a$ and since $a$ is a monomorphism $s=\lambda u$. Similarly $t=\lambda v$ which proves that $k=a \cap b$. We remark that by the above theorem the category of regular monomorphisms into $X$ has finite products.

Definition 10. If $b \subseteq Y$ and $X \stackrel{f}{\rightarrow} Y$ then an inverse image of $b$ through $f$ is a mapping $A \stackrel{a}{\rightarrow} X$ with the property that there exists a mapping $c$ such that the diagram below is a pull-back diagram.

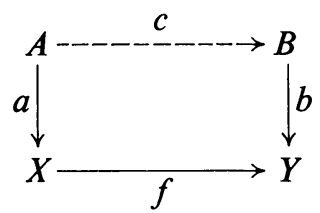

Whenever $a$ is an inverse image of $b$, we write $a=f^{-1}(b)$.

THEOREM 3. The inverse image of a regular monomorphism is a regular monomorphism. In fact, if $b=\mathrm{Eq}(u, v)$ then $f^{-1}(b)=\mathrm{Eq}(f u, f v)$.

Proof. Let $a=f^{-1}(b)$. Since $b$ is a monomorphism $a$ is a monomorphism. We shall prove that $a=\mathrm{Eq}(f u, f v)$. Clearly $a f u=c b u=c b v=a f v$. Suppose $\lambda$ is a mapping such that $\lambda f u=\lambda f v$. Since $b=\operatorname{Eq}(u, v)$, there exists a mapping $\mu$ for which $\lambda f=\mu b$. This implies the existence of a unique $\theta$ with the properties $\lambda=\theta a$ and $\mu=\theta c$.

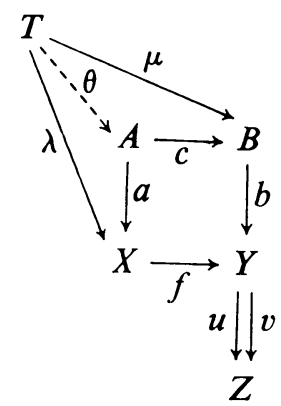


It follows that $a=\mathrm{Eq}(f u, f v)$. Q.E.D.

Proposition 14 (a). Suppose $a \subseteq X, b$ is a monomorphism with codomain $X$ and $|b| \subseteq|a|$. Then there exists a mapping $u$ such that $b=u a$.

(b) If $b \subseteq X$ and $a \subseteq X$, then $b \subseteq a$ if and only if, for every $x \in X, x \in b$ implies $x \in a$.

Proof. (a) Since $a \subseteq X$ there exists $f, g$ such that $a=\operatorname{Eq}(f, g)$. Since $|b| \subseteq|a|$ there exists a mapping $d$ with the property $|b|=d|a|$. Therefore $|b f|=|b||f|$ $=d|a||f|=d|a f|$ and $|b g|=|b||g|=d|a||g|=d|a g|$. But $a f=a g$ and hence $|b f|=|b g|$. By the faithfulness of $|\quad|$ we must have $b f=b g$ and hence there exists a map $a$ such that $b=u a$.

(b) We first remark that this proposition is a theorem in Lawvere's system and hence by the Theorem Schema, for discrete spaces it is also provable from our axioms. Suppose that for every $x \in X$, if $x \in b$ then $x \in a$. Then for every $x \in|X|$, $x \in|b|$ implies $x \in|a|$. By the above remark we must have $|b| \subseteq|a|$ which together with (a) implies the existence of a map $u$ such that $b=u a$. The opposite implication is obvious.

We shall next define "open subspaces" in our system. The notion of open set is primitive in topology. In order to recapture this notion in our system, we shall enrich our language by adding to it a new undefined term, namely an individual constant which we shall denote by $o$.

Definition 11. $E$ and 1 are respectively the codomain and the domain of the mapping $o$.

Assumption 1 was needed in order to define the object 1 . With the above definition, in order to dispense with this assumption, we have to state that 1 is a terminal object. We include this in the next axiom.

Axıом 8. 1 is a terminal object and $E$ has exactly three endomorphisms.

$\boldsymbol{T}$ is a model of the above axiom because we can interpret $E$ as a space with two points $o, 1$ and three open sets (the empty set and the sets $\{o\}$ and $\{o, 1\})$. Clearly this space has exactly three endomorphisms, i.e. the identity and the two constant mappings. We shall interpret $o$ as the open point of this space.

Proposition 15. The object E has two elements.

Proof. Clearly $E \neq 0, E \neq 1$ and hence $E$ has at least two elements. It follows from Proposition 4 that two different elements of $E$ generate different constant endomorphisms of $E$. Also if $t$ is the mapping from $E$ into 1 , then $t x \neq E$ for all $x \in E$ (otherwise $E=1$ ). This together with the fact that $E$ has only three endomorphisms implies that $E$ has exactly two elements. Q.E.D.

By the above proposition, Assumption 2 follows from Axiom 8.

We shall denote by $c$ the element of $E$ different from $o\left({ }^{5}\right)$. In $T$ there is a one-to-

(5) We no longer need a labeling for the elements of 2 since they can be defined $i_{1}=|o|$ and $i_{0}=|c|$. 
one correspondence between the open subsets of a topological space $X$ and the mapping $X \rightarrow E$ namely, for every open subset $A$ of $X$ we consider the mapping

$$
\begin{array}{rlr}
\psi_{A}(x)=o & \text { if } x \in A, \\
=1 & \text { if } x \notin A .
\end{array}
$$

Clearly the correspondence $A \rightarrow \psi_{A}$ is one-to-one. It is also onto because if $X \stackrel{\psi}{\rightarrow} E$ is a continuous mapping then $A=\psi^{-1}(o)$ is open and $\psi_{A}=\psi$. This shows that the following definition of "open subspaces" is adequate.

Definition 12. A subspace $a$ of $X$ is called open if there exists a mapping $X \stackrel{\text { }_{a}}{\rightarrow} E$ such that $a=\mathrm{Eq}\left(\psi_{a}, X \rightarrow 1 \stackrel{\circ}{\rightarrow} E\right)$. (We remark that if there exists a $\psi_{a}$ with this property then $\psi_{a}$ is unique.)

Clearly the mappings $0 \rightarrow X$ and $X$ are open subspaces of $X$ and $o$ is an open subspace of $E$.

Proposition 16. For every mapping $X \stackrel{f}{\rightarrow} Y$, if $b$ is an open subspace of $Y$, then the inverse image of $b$ through $f$ is an open subspace of $X$.

Proof. Since $b$ is open in $Y$ there exists a mapping $\psi_{b}$ such that

$$
b=\operatorname{Eq}\left(\psi_{b}, Y \longrightarrow 1 \stackrel{o}{\longrightarrow} E\right) .
$$

Let $a=f^{-1}(b)$ and $\psi_{a}=f \psi_{b}$. Then by Theorem 6 ,

$$
a=\mathrm{Eq}\left(f \psi_{b}, X \stackrel{f}{\longrightarrow} Y \stackrel{o}{\longrightarrow} 1 \stackrel{o}{\longrightarrow} E\right)=\mathrm{Eq}\left(\psi_{a}, X \longrightarrow 1 \stackrel{o}{\longrightarrow} E\right)
$$

and hence $a$ is open. Q.E.D.

Proposition 17. Every monomorphism with discrete codomain $D$ is an open subspace of $D$.

Proof. Let $a \subseteq D$. By Proposition 12, $a$ has a characteristic function $\phi_{a}$, i.e. $a=\mathrm{Eq}\left(\phi_{a}, D \rightarrow 1 \stackrel{i_{1}}{\rightarrow} 2\right)$. It follows that $a=\phi_{a}^{-1}\left(i_{1}\right)$. But $i_{1}=t_{E}^{-1}(o)$ and hence by Proposition $16 i_{1}$ and therefore $a$ are open.

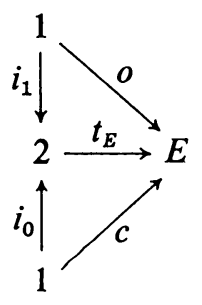

We have shown that every mapping is "continuous". We need an axiom which will guarantee that we have enough "continuous" mappings. Since we may think of | as being a forgetful functor built into the system, any mapping $|X| \stackrel{d}{\rightarrow}|Y|$ may be interpreted as a set-mapping. Supposing $d$ is "continuous" with respect to 
$X$ and $Y, d$ must be the set-mapping of some map $X \stackrel{f}{\rightarrow} Y$, i.e. $d=|f|$. This can be formulated as follows:

"Let $|X| \stackrel{d}{\rightarrow}|Y|$ be a mapping with the property that whenever $b$ is an open subspace of $Y$ and $a$ is a subspace of $X$ such that $d^{-1}(|b|)=|a|, a$ is also open. Then there exists a mapping $X \stackrel{f}{\rightarrow} Y$ such that $d=|f| . "$ It is easy to see that the above can be rephrased, by using mappings into $E$ instead of open subspaces, as follows:

Axiom 9. Let $|X| \stackrel{d}{\rightarrow}|Y|$ be a mapping such that for every map $Y \stackrel{b}{\rightarrow} E$ there exists a map $X \stackrel{a}{\rightarrow} E$ for which the diagram below commutes.

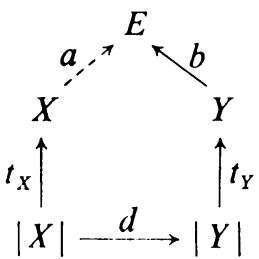

Then there exists a mapping $X \stackrel{f}{\rightarrow} Y$ such that $d=|f|$.

We note that such a map is unique.

Definition 13. An indiscrete space $X$ is an object for which the only maps from $X$ to $E$ are the constant maps, i.e. $X \rightarrow 1 \stackrel{o}{\rightarrow} E, X \rightarrow 1 \stackrel{c}{\rightarrow} E$.

Clearly 1 is an indiscrete space but so far nothing guarantees the existence of other indiscrete spaces.

Axiom 10. For every discrete space $D$ there exists an indiscrete space $\bar{D}$ such that $|\bar{D}|=D$.

We note that the object $\bar{D}$ is determined up to isomorphism and that the operation " - " is functorial.

We shall denote by $J$ the indiscrete space associated to 2 , i.e. $J=\overline{2}$.

Proposition 18. “| |" is a left adjoint to “-"”.

Proof. By Axiom 9 for every object $X$, discrete space $D$ and map $|X| \stackrel{f}{\rightarrow} D$ there exists a unique map $X \stackrel{f}{\rightarrow} \bar{D}$ such that $|\bar{f}|=f$.

COROLlaRY. | | preserves coproducts and coequalizers.

PROPOSITION 19. If $q$ is the regular coimage of $f, q^{*}$ is the regular image of $f$ and $h$ is the unique mapping such that $f=q h q^{*}$, then $h$ is a bijection.

Proof. | preserves regular images and coimages and hence by Proposition 11, $|h|$ is an isomorphism. By Proposition 7, $h$ is a bijection.

COROLlary. If $m$ is an extremal monomorphism then $m$ is regular.

Proof. By the above proposition $m=\lambda q^{*}$ where $\lambda$ is an epimorphism. Since $m$ is extremal, $\lambda$ is an isomorphism and hence $m$ is regular. So the extremal monomorphisms coincide with the regular ones. 
Actually, for every subspace $a \subseteq X$ we have a map $X_{\stackrel{\phi_{a}}{\rightarrow}}^{\rightarrow}(J=\overline{2})$ such that $a=\mathrm{Eq}\left(\phi_{a}, X \rightarrow 1 \stackrel{i_{1}}{\rightarrow} J\right)$. Indeed, the characteristic function of $|a|$ can be lifted to a mapping $X \stackrel{\phi_{a}}{\rightarrow} J$ with the above property. The correspondence $a \rightarrow \phi_{a}$ is onto and if $a \nsubseteq b$ then $\phi_{a} \neq \phi_{b}$. Hence we may identify the subspaces of $X$ with mappings $X \rightarrow J$. The mappings from $X$ to $J$ have a boolean algebra structure.

Since the open subspaces of $X$ are in a one-to-one correspondence with the mappings $X \rightarrow E$, we must make sure that we have enough such mappings to guarantee the properties satisfied by the open subsets of a topological space. For instance, the intersection of two arbitrary open subsets must be open. By postulating the existence of some mapping of $E \times E$ into $E$, the above property can be proved in the resulting system of axioms.

The following observation motivates our next axiom. Consider the product $E \times E, p_{1}, p_{2}$. In $T$ the interpretation of $E \times E$ is the product space. The open subsets of this space are: the empty set, the total set, $p_{1}^{-1}(o)=\{(o, o),(o, 1)\}, p_{2}^{-1}(o)$ $=\{(o, o),(1, o)\}$ and the set $p_{1}^{-1}(o) \cap p_{2}^{-1}(o)=\{(o, o)\}$. Since $E \times E$ must have five open subspaces, we should have five mappings from $E \times E$ into $E$. The axioms stated so far guarantee the existence of only four such mappings, i.e.

$$
E \times E \underset{p_{2}}{\stackrel{p_{1}}{\longrightarrow}} E \text { and } E \times E \longrightarrow 1 \underset{c}{\stackrel{o}{\longrightarrow}} E .
$$

We need an additional mapping corresponding to the open set $p_{1}^{-1}(o) \cap p_{2}^{-1}(o)$ $=\{(o, o)\}$. For this reason we add the following axiom.

Axıом 11. The mapping

$$
1 \stackrel{\langle o, o\rangle}{\longrightarrow} E \times E
$$

is an open subspace of $E \times E$.

We denote by $\wedge_{E}$ the map into $E$ corresponding to $\langle o, o\rangle$, i.e. $\langle o, o\rangle=$ $\mathrm{Eq}\left(\wedge_{E}, E \times E \rightarrow 1 \stackrel{\circ}{\rightarrow} E\right)$. Clearly $\left|\wedge_{E}\right|=\wedge$.

We define the intersection of the mappings $X \stackrel{\Psi}{\rightarrow} E$ and $X \stackrel{\psi^{\prime}}{\rightarrow} E$ by the formula: $\psi \wedge \psi^{\prime}=\left\langle\psi, \psi^{\prime}\right\rangle \wedge_{E}$. This operation is associative, commutative and $\psi \wedge \psi=\psi$. Hence we have an order relation on the morphisms from $X$ to $E, \wedge$ is the greatest lower bound operation and thus the mappings from $X$ to $E$ form a category with products of two objects.

THEOREM 4. The intersection of two open subspaces $a, b \subseteq X$ is an open subspace of $X$. In fact $a \cap b=\mathrm{Eq}\left(\psi_{a} \wedge \psi_{b}, X \rightarrow 1 \stackrel{o}{\rightarrow} E\right)$ where $\psi_{a}, \psi_{b}$ are the maps into $E$ associated to $a, b$.

Proof. By hypothesis $a=\mathrm{Eq}\left(\psi_{a}, X_{\stackrel{t}{\rightarrow}}^{\rightarrow} \stackrel{\circ}{\rightarrow} E\right)$ and $b=\mathrm{Eq}\left(\psi_{b}, X_{\stackrel{t}{\rightarrow}}^{\rightarrow} \stackrel{\circ}{\rightarrow} E\right)$. This together with Theorem 3 implies $a \cap b=\mathrm{Eq}\left(\left\langle\psi_{a}, \psi_{b}\right\rangle, t\langle o, o\rangle\right)$. We must show that $a \cap b$ is open hence we seek a map $X \stackrel{\psi_{a} \cap_{b}}{\longrightarrow} E$ such that

$$
a \cap b=\operatorname{Eq}\left(\psi_{a \cap b}, X \stackrel{t}{\longrightarrow} 1 \stackrel{o}{\longrightarrow} E\right) .
$$


Using the fact that $\langle o, o\rangle=\operatorname{Eq}\left(\wedge_{E}, E \times E \stackrel{t^{\prime}}{\rightarrow} 1 \stackrel{o}{\rightarrow} E\right)$ and the above formula for $a \cap b$ it is easy to verify that $\psi_{a \cap b}=\left\langle\psi_{a}, \psi_{b}\right\rangle \wedge_{E}=\psi_{a} \wedge \psi_{b}$ has the required property.

In $T$ the interior of a subset $A$ of $X$ is the largest open subset of $X$ contained in $A$. The next axiom asserts the existence of this set.

Axiom 12. For every $a \subseteq X$ there exists an open subspace $a^{o}$ of $X$ such that:

(i) $a^{\circ} \subseteq a$,

(ii) for every open subspace $b$ of $X$ such that $b \subseteq a$, we have $b \subseteq a^{o}$.

Clearly $a^{o}$ is determined up to isomorphism. If $a$ is open we may write $a^{o}=a$. Consequently $\left(a^{o}\right)^{o}=a^{o}$ and $X^{o}=X$. To show that " $o$ " is an "interior operation" it remains to prove that it is distributive with respect to intersection. This is an immediate consequence of Axiom 12 by which " $o$ " is a right adjoint of the forgetful functor which takes open subspaces into subspaces of $X$.

Using maps $X \rightarrow J$ instead of subspaces $a \subseteq X$ and the map $E \stackrel{\varepsilon}{\rightarrow} J$ obtained by lifting the identity on 2 we can restate Axiom 12 as follows:

Axiom $12^{\prime}$. For each object $X$, composition of the maps from $X$ to $E$ with $\varepsilon$ has a right adjoint.

We denote by $\psi^{\circ}$ the map associated to the map $X \stackrel{\Downarrow}{\rightarrow} J$ by this adjoint.

Another way of restating Axiom 12 is by saying that the functor $\mid$ from the maps $X \rightarrow E$ into the maps $|X| \rightarrow 2$ admits a right adjoint.

We recall that every monomorphism $m$ with discrete codomain $D$ has a characteristic function $D \stackrel{\phi_{m}}{\rightarrow}$ 2, i.e. $m=\mathrm{Eq}\left(\phi_{m}, D \rightarrow 1 \stackrel{i_{1}}{\rightarrow}\right.$ 2). By the exponentiation axiom there exists a unique mapping $\phi_{m}^{*}$ such that the diagram below commutes.

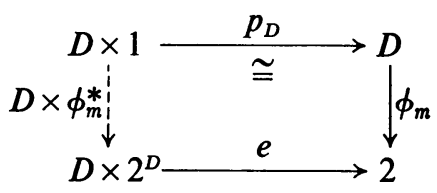

We shall use the notation $m^{*}=\left|\phi_{m}^{*}\right| \in\left|2^{D}\right|$ and we extend this operation for subspaces $a \subseteq X$ by defining $a^{*}=|a|^{*}$.

If $f$ is a mapping with discrete domain $D$ and discrete codomain $D^{\prime}$ and $m$ is a monomorphism into $D^{\prime}$ then $m^{*}\left|2^{f}\right|=\left(f^{-1}(m)\right)^{*}$.

DEFINITION 14. If $D$ is a discrete space, a mapping $\left|2^{D}\right| \stackrel{I}{\rightarrow}\left|2^{D}\right|$ is called an interior operation on $D$ if :

(i) $I^{2}=I\left(I^{2}=I I\right)$;

(ii) $D^{*} I=D^{*}$;

(iii) for every $x \in\left|2^{D}\right|, x I \cap x=x I$;

(iv) for every pair $x, y \in\left|2^{D}\right|,(x \cap y) I=x I \cap y I$.

Clearly (iii) and (iv) can be restated without using elements as follows:

(iii)' $\left\langle I,\left|2^{D}\right|\right\rangle\left|\wedge^{D}\right|=I$; and (iv) $\left|\wedge^{D}\right| I=I \times I\left|\wedge^{D}\right|$.

THEOREM 5. For every object $X$, there exists an interior operation $\left|2^{D}\right| \stackrel{I}{\rightarrow}\left|2^{D}\right|$ such that $D=|X|$ and $a \subseteq X$ is open if and only if $a^{*} I=\left(a^{o}\right)^{*}$. 
Proof. It suffices to construct $I$ such that $a^{*} I=\left(a^{0}\right)^{*}$.

Consider the evaluation map $D \times 2^{D} \stackrel{e}{\rightarrow} 2$. The map $D \times\left|2^{D}\right| \stackrel{|e|}{\rightarrow} 2$ is the evaluation map for discrete spaces. Consider the map $X \times\left|2^{D}\right| \stackrel{e^{\circ}}{\rightarrow} E$ associated to $|e|$ by Axiom 12. Clearly $\left|e^{o}\right| \subset|e|$. Let $I$ denote the unique map such that the diagram below is commutative.

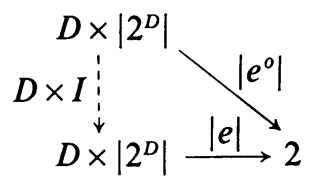

We have to show that for every $a \subseteq X$ we have $a^{*} I=\left(a^{o}\right)^{*}$. Let $v$ be a subspace of $X$ such that $v^{*}=a^{*} I$. Then

$v=\mathrm{Eq}\left(X \stackrel{\cong}{\cong} X \times 1 \stackrel{X \times a^{*}}{\longrightarrow} X \times\left|2^{D}\right| \stackrel{e^{o}}{\longrightarrow} E, X \longrightarrow 1 \stackrel{o}{\longrightarrow} E\right)$.

For this it suffices to show that $x \in v$ if and only if $\left\langle x, a^{*}\right\rangle e^{o}=o$ which follows easily from the definition of $v$. Hence $v$ is open. Using the above condition for $x \in v$ and the fact that $\left|e^{o}\right| \subset|e|$ we deduce that $x \in a$. In order to prove that $v=a^{o}$ it remains to show that $a^{o} \subseteq v$ or equivalently that $x \in a^{o}$ entails $\left\langle x, a^{*}\right\rangle e^{o}=o$. To see this consider the map $X \stackrel{\psi_{\left(a^{0}\right)}}{\longrightarrow} E$ associated to $a^{0}$ and the characteristic function $\left|2^{D}\right| \stackrel{\phi_{a}^{*}}{\longrightarrow} 2$ of $1 \stackrel{a^{*}}{\rightarrow}\left|2^{D}\right|$. The map $\lambda=\left(\psi_{\left(a^{o}\right)} \times \phi_{a^{*}} i\right) \wedge_{E}$, where $2 \stackrel{i}{\rightarrow} E$ such that $i_{1} i=o$, $i_{0} i=c$, determines an open set and since $a^{o} \subseteq a$, it is easy to show that $|\lambda| \subset|e|$. Therefore $\lambda \subset e^{o}$ by adjointness. If $x \in a^{o}$ then $\left\langle x, a^{*}\right\rangle \lambda=\left\langle x, a^{*}\right\rangle\left(\psi_{\left(a^{o}\right)} \times \phi_{a}, i\right) \wedge_{E}=o$. Since $\lambda \subset e^{o},\left\langle x, a^{*}\right\rangle e^{o}=o$ and hence $x \in v$.

We have proved that to every $X$ there corresponds an interior operation on $D=|X|$. Our last axiom says that every interior operation can be obtained in this way.

Axıом 13. For every discrete space $D$ and every interior operation $I$ on $D$, there exists an object $X$ such that

(i) $|X|=D$,

(ii) for every subspace $a$ of $X, a^{*} I=\left(a^{o}\right)^{*}$.

We remark that the object $X$ whose existence is stated in the above axiom is determined by $I$ up to isomorphism. Indeed, if $X$ and $Y$ are two objects corresponding to $I$, the identity $D=|X|=|Y|$ may be lifted by Axiom 9 to a map $X \stackrel{f}{\rightarrow} Y$ which has an inverse.

If $X$ is an object corresponding to $I$ by the above axiom, then $a$ is an open subspace of $X$ if and only if $a^{*} I=a^{*}$.

4. Metatheorems. In this section we show that the elementary system of axioms which we have constructed, together with one nonelementary axiom form a characterization of $\boldsymbol{T}$. The proof is informal but it could be formalized within a sufficiently strong set theory. 
Our discussion will be restricted to locally small categories, i.e. to categories with the property that for every pair of objects $A$ and $B$, the class of mappings from $A$ to $B$ is a set.

Suppose $D$ is a category satisfying Lawvere's elementary axioms for $\boldsymbol{S}$. We shall denote by $\boldsymbol{T}_{\boldsymbol{D}}$ the category whose objects are ordered pairs consisting of an object $D$ of $D$ and an interior operation $\left(^{6}\right) I$ on $D$ and whose morphisms are 5-tuples $\left\langle D, I ; f ; D^{\prime}, I^{\prime}\right\rangle$ where $D \stackrel{f}{\rightarrow} D^{\prime}$ is a map in $D$ satisfying the continuity conditions with respect to $I, I^{\prime}$. I.e. for every $x \in 2^{D^{\prime}}$ such that $x I^{\prime}=x$ we have $x 2^{f} I=x 2^{f}$ or equivalently $I^{\prime} 2^{f} I=I^{\prime} 2^{f} . T_{D}$ is the category of topological spaces in Lawvere's system.

Metatheorem 1A. Let $\boldsymbol{C}$ be a category, model of the thirteen axioms. Let $\boldsymbol{D}$ be the full subcategory of discrete spaces. Then $\mathbf{D}$ is a model of Lawvere's axioms for the category of sets and $C$ is equivalent with $\boldsymbol{T}_{\boldsymbol{D}}$.

Proof. We define a functor $\stackrel{F}{\rightarrow} \boldsymbol{T}_{D}$ as follows: $F(X)=(D, I)$ where $D=|X|$ and $I$ is the interior operation on $D$ associated to $X$ by Theorem 5 . If $X \stackrel{f}{\rightarrow} Y$ is a map in $C$ and $F(X)=(D, I), F(Y)=\left(D^{\prime}, I^{\prime}\right)$, then $F(f)=\left(D, I ;|f| ; D^{\prime}, I^{\prime}\right)$ is a map in $\boldsymbol{T}_{\boldsymbol{D}}$.

To see this, consider $y \subseteq Y$ and let $x=f^{-1}\left(y^{o}\right)$. Since $y^{o}$ is open in $Y$, by Proposition $16, x$ is open in $X$ and hence $x^{*} I=x^{*}$. The functor $\mid$ preserves inverse images, so we have $|f|^{-1}\left(\left|y^{o}\right|\right)=|x|$ and hence $y^{*} I^{\prime}\left|2^{|f|}\right|=\left(y^{o}\right)^{*}\left|2^{|f|}\right|=x^{*}$. Therefore $y^{*} I^{\prime}\left|2^{|f|}\right| I=x^{*} I=x^{*}=y^{*} I^{\prime}\left|2^{|f|}\right|$. This equality holds for all $y^{*} \in 2^{D^{\prime}}$ and hence $I^{\prime} 2^{|f|} I=I^{\prime} 2^{|f|}$, i.e. $F(f) \in T_{D}$. Clearly $F: C \rightarrow T_{D}$ is a functor. We shall now define a functor $G: T_{D} \rightarrow C$. For this consider $(D, I) \in T_{D}$ and let $X$ be the object assigned by Axiom 13 to the pair $(D, I)$. ( $X$ is uniquely determined up to isomorphism.) We define $G(D, I)=X$. If $D \stackrel{d}{\rightarrow} D^{\prime}$ is a morphism in $T_{D}$ from $(D, I)$ into $\left(D^{\prime}, I^{\prime}\right)$ and $X=G(D, I), Y=G\left(D^{\prime}, I^{\prime}\right)$, then $d$ satisfies the condition in Axiom 9 with respect to $X$ and $Y$. In order to prove this we first note that $|X|=D,|Y|=D^{\prime}$. Furthermore, if $v$ is an open subspace of $Y$, then clearly $v^{*} I^{\prime}=v^{*}$. Suppose $u$ is a subspace of $X$ such that $d^{-1}(|v|)=|u|$. Then, since $d \in T_{D}$, we have $v^{*}\left|2^{d}\right| I=v^{*}\left|2^{d}\right|$ $=u^{*}$ and hence $u^{*} I=v^{*}\left|2^{d}\right| I^{2}=v^{*}\left|2^{d}\right|=u^{*}$. This implies that $u$ is open in $X$. According to Axiom 9 there exists a (unique) mapping $X \stackrel{f}{\rightarrow} Y$ such that $|f|=d$. Let $G(d)=f$. Clearly $G$ is a functor. Furthermore, $F \circ G$ and $G \circ F$ are naturally equivalent to the identities on $T_{D}$ and $C$ respectively. Indeed if $X=G(D, I)$, then for every $x \subseteq X$ we have $x^{*} I=\left(x^{0}\right)^{*}$, and hence $F(X)=(D, I)$. Therefore $F \circ G(D, I)$ $=(D, I)$ for all $(D, I)$, and $F \circ G=T_{D}$. Now suppose $(D, I)=F(X)$. Clearly for all $x \subseteq X$, we have $x^{*} I=\left(x^{0}\right)^{*}$. Let $G(D, I)=Y$. Then $|Y|=D$ and $y^{*} I=\left(y^{0}\right)^{*}$. Since both $X$ and $Y=G \circ F(X)$ satisfy Axiom 13 with respect to the pair $(D, I)$, we must have $G \circ F(X) \cong X$ and this isomorphism is natural. Therefore, $F: C \rightarrow T_{D}$ is an equivalence of categories.

${ }^{(6)}$ The notion of interior operation makes sense in any category $D$ model of Lawvere's axioms. 
We have proved that from a model $\boldsymbol{C}$ of the thirteen axioms we obtain a model $\boldsymbol{D}$ of Lawvere's axioms and the topological spaces over $\boldsymbol{D}$ form a category $\boldsymbol{T}_{\boldsymbol{D}}$ equivalent to $\boldsymbol{C}$. The natural question which arises now is whether starting with a model $\boldsymbol{D}$ of Lawvere's axioms, the category $\boldsymbol{T}_{\boldsymbol{D}}$ of topological spaces over $\boldsymbol{D}$ is a model of the thirteen axioms. This is indeed true but the proof is in part tedious as in the case of proving the existence of products which involves the construction of an interior operation on the product set by using interior operations on the factors. This only shows that it is better to assume such basic properties from the beginning. The remaining part of the next metatheorem is immediate.

Metatheorem 1B. Let $\boldsymbol{L}$ be a model of Lawvere's axioms. Then $\boldsymbol{T}_{L}$ is a model of the thirteen axioms. If $\boldsymbol{D}$ is the full subcategory of discrete spaces of $\boldsymbol{T}_{\boldsymbol{L}}$ then $\boldsymbol{L}$ is isomorphic with $\boldsymbol{D}$.

We emphasize the fact that in the Metatheorem 1 only elementary axioms were involved and no set-theoretical requirement was needed. In principle one could develop topology by starting from these purely categorical axioms.

METATHEOREM 2. Let $\boldsymbol{D}$ be any locally small category, model of Lawvere's axioms. If $\boldsymbol{D}$ is complete then $\boldsymbol{T}_{\boldsymbol{D}}$ is equivalent to $\boldsymbol{T}$.

Proof. By Lawvere's metatheorem $\boldsymbol{D}$ is equivalent to the category of sets and the functor which sets up this equivalence is $H^{1}: D \rightarrow S$ where $H^{1}(D)=$ hom $(1, D)$ $=\{x \mid x \in D\}$. Since an equivalence preserves exponentiation, for any object $D$ of $D, H^{1}\left(2^{D}\right) \cong 2^{H^{1}(D)}$. If $(D, I)$ is an object of $\boldsymbol{T}_{D}$ then $H^{1}(I)$ determines an interior operation on $H^{1}(D)$ since all the equations involved are preserved. This defines a topology on $H^{1}(D)$. Let $F(D, I)$ be this topological space. Then $F$ is functorial. The fact that $H^{1}$ is an equivalence implies that also $F$ is an equivalence.

COROllaRy. Let $\boldsymbol{C}$ be a complete locally small category, model of the thirteen axioms. Then $C$ is equivalent to $\boldsymbol{T}$.

Proof. By the first metatheorem $C$ is equivalent to $\boldsymbol{T}_{\boldsymbol{D}}$. Since $\boldsymbol{C}$ is complete and the functor || is limit and colimit preserving, $D$ is complete. By the Theorem Schema $\boldsymbol{D}$ is also a model of Lawvere's axioms. Hence, according to the second metatheorem $\boldsymbol{T}_{\boldsymbol{D}}$ is equivalent to $\boldsymbol{T}$.

Thus the three constructions of the topological category, i.e. the traditional construction, the translation of this construction in Lawvere's system and our axiomatization lead essentially to the same thing.

In this last part of the paper we investigate what can be obtained by using the first 12 axioms. The following metatheorem indicates this as well as a different condition for equivalence.

MetATHEOREM 3. Let $C$ be a complete locally small category, model of the first twelve axioms. Then there exists a full imbedding $\boldsymbol{C} \underset{\boldsymbol{F}}{\rightarrow} \boldsymbol{T}$. If $\boldsymbol{D}$ is the full discrete 
subcategory of $\boldsymbol{C}$ and $\boldsymbol{D T}$ is the category of discrete topological spaces then the restriction of $F$ on $D$ is an equivalence between $D$ and DT. Furthermore F preserves the object $E$ as well as the indiscrete spaces. If $F$ is limit preserving then $F$ is an equivalence.

Proof. If $C$ is an object of $C$ and $a \subseteq C$ we consider $V_{a}=\{x \in C \mid x \in a\}$. Then $\tau=\left\{V_{a} \mid a \subseteq C, a^{o}=a\right\}$ defines a topology on $H^{1}(C)=\{x \mid x \in C\}$. Let $F(C)=$ $\left(H^{1}(C), \tau\right)$. If $C \stackrel{f}{\rightarrow} C^{\prime}$ then $H^{1}(f)$ is continuous and if $F(f)=H^{1}(f), F$ is a functor. Since $H^{1}$ is faithful, so is $F$. $F$ is also full. To see this suppose $F(C) \stackrel{\lambda}{\rightarrow} F\left(C^{\prime}\right)$ is a map in $T$. Then $H^{1}(C) \stackrel{\lambda}{\rightarrow} H^{1}\left(C^{\prime}\right)$ determines a map $H^{1}(|C|) \stackrel{\mu}{\rightarrow} H^{1}\left(\left|C^{\prime}\right|\right)$ because $t_{C}, t_{C^{\prime}}$ are bijections. Since $H^{1}: D \rightarrow S$ is an equivalence $H^{1}$ is full, i.e. $\mu=H^{1}(d)$ with $|C| \stackrel{d}{\rightarrow}\left|C^{\prime}\right|$. It can be shown that $d$ satisfies the condition in Axiom 9 and hence there is a mapping $C \stackrel{f}{\rightarrow} C^{\prime}$ such that $|f|=d$. It is easy to see that $F(f)=\lambda$. Hence $F$ is a full imbedding. To end the proof we only need to show that if $F$ is limit preserving then $F$ is representative, i.e. for every topological space $X$ there exists an object $A$ of $C$ with the property that $F(A) \cong X$. For this we use the following proposition, noticed by $\mathrm{S}$. Baron, which follows easily from the imbedding lemma [6]:

"Every topological space can be imbedded in a product of copies of the indiscrete space with two points and the topological space with two points and three open subsets."

Clearly $F(E)$ is a space with two points and three open subsets and $F(J)$ is an indiscrete space with two points. If $X$ is a topological space then there exists an imbedding $X \rightarrow \prod_{\lambda \in \Lambda} X_{\lambda}$ where $X_{\lambda}$ is $F(E)$ or $F(J)$ for all $\lambda \in \Lambda$. Since $F$ preserves products $\prod_{\lambda \in \Lambda} X_{\lambda} \cong F\left(\prod_{\lambda \in \Lambda} E_{\lambda}\right)$ where $E_{\lambda}$ is $E$ or $J$ depending on whether $X_{\lambda}$ is $F(E)$ or $F(J)$.

The proof will be completed once we show that for every subspace $X$ of a topological space $F(C)$, there exists a subspace $a$ of $C$ such that $X$ is the image of $F(a)$ in $F(C)$.

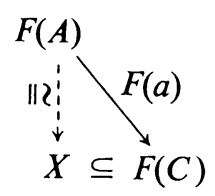

This follows from the fact that every subset of $H^{1}(C)$ determines a subset of $H^{1}(|C|)$ which in turn is determined by a monomorphism into $|C|$. Clearly to every such monomorphism there corresponds a subspace $a$ of $C$ which has the required property.

\section{BIBLIOGRAPHY}

1. R. F. Arens, A topology for spaces of transformations, Ann. of Math. (2) 47 (1946), 480-495. MR 8, 165.

2. S. Eilenberg and Saunders MacLane, General theory of natural equivalences, Trans. Amer. Math. Soc. 58 (1945), 231-294. MR 7, 109. 
3. R. H. Fox, On topologies for function spaces, Bull. Amer. Math. Soc. 51 (1945), 429-432. MR 6, 278.

4. P. Freyd, Abelian categories. An introduction to the theory of functions, Harper's Series in Modern Math., Harper \& Row, New York, 1964. MR 29 \#3517.

5. J. R. Isbell, Subobjects, adequacy, completeness and categories of algebras, Rozprawy Mat. 36 (1964). MR 29 \#1238.

6. J. L. Kelley, General topology, Van Nostrand, Princeton, N. J., 1955. MR 16, 1136.

7. F. W. Lawvere, Functorial semantics of algebraic theories, Dissertation, Columbia University, New York, 1963.

8. - An elementary theory of the category of sets, Proc. Nat. Acad. Sci. U.S.A. 52 (1964), 1506-1511. MR 30 \#3025.

9. - The category of categories as a foundation for mathematics, Proc. Conf. Categorical Algebra, (La Jolla, Calif., 1965), Springer, New York, 1966. MR 34 \#7332.

10. Saunders MacLane, Categorical algebra, Bull. Amer. Math. Soc. 71 (1965), 40-106. MR 30 \#2053.

11. B. Mitchell, Theory of categories, Pure and Appl. Math., vol. 17, Academic Press, New York, 1965. MR 34 \#2647.

12. D. Schlomiuk, A characterization of the category of topological spaces, Notices Amer. Math. Soc. 14 (1967), 269. Abstract \#67T-180.

13. E. Spanier, Quasi-topologies, Duke Math. J. 30 (1963), 1-14. MR 26 \#1847.

UNIVERSITÉ DE MONTRÉAL,

Montréal, Quebec, Canada 